\title{
Predicting Mycobacterium tuberculosis in patients with community-acquired pneumonia
}

\author{
Rodrigo Cavallazzi', Timothy Wiemken², Diana Christensen², Paula Peyrani², \\ Francesco Blasi ${ }^{3}$, Gur Levy ${ }^{4}$, Stefano Aliberti ${ }^{5}$, Robert Kelley ${ }^{2}$, Julio Ramirez ${ }^{2}$ and \\ the Community-Acquired Pneumonia Organization (CAPO) Investigators
}

\author{
Affiliations: \\ 'Division of Pulmonary, Critical Care and Sleep Disorders Medicine, University of Louisville, Louisville, KY, and \\ ${ }^{2}$ Division of Infectious Diseases, University of Louisville, Louisville, KY, USA. \\ ${ }^{3}$ Dipartimento di Fisiopatologia Medico-Chirurgica e dei Trapianti, University of Milan, IRCCS Fondazione Cà \\ Granda, Ospedale Maggiore Policlinico, Milan, and \\ ${ }^{5}$ Dipartimento di Medicina Clinica e Prevenzione, University of Milan-Bicocca, San Gerardo Hospital, Monza, \\ Italy. \\ ${ }^{4}$ Dept of Pulmonology, Hospital Universitario de Caracas, Central University of Venezuela, Caracas, Venezuela.
}

Correspondence: R. Cavallazzi, Division of Pulmonary, Critical Care and Sleep Disorders Medicine, University of Louisville, Ambulatory Care Building, 550 S. Jackson Street, Louisville, KY, 40202, USA. E-mail: rcavallazzidagmail.com

ABSTRACT The 22 risk factors suggested by the Centers for Disease Control and Prevention (CDC) to predict patients at risk for Mycobacterium tuberculosis have not been evaluated in hospitalised patients with community-acquired pneumonia (CAP). We evaluated which of the CDC risk factors best predict M. tuberculosis in these patients. To our knowledge, this is the first time a score has been developed assessing these risk factors.

This was a secondary analysis of 6976 patients hospitalised with CAP enrolled in the CommunityAcquired Pneumonia Organization International Cohort Study. Using Poisson regression, we selected the subset of risk factors that best predicted the presence of CAP due to M. tuberculosis. This subset was compared to the CDC risk factors using receiver operating characteristic (ROC) curve analysis.

Five risk factors were found to best predict CAP due to M. tuberculosis: night sweats, haemoptysis, weight loss, M. tuberculosis exposure and upper lobe infiltrate. The area under the ROC curve for all CDC risk factors was $71 \%$ and $89 \%$ for the subset of five risk factors.

The CDC-suggested risk factors are poor at predicting the presence of M. tuberculosis in hospitalised patients with CAP. With a subset of five risk factors identified in this study, we developed a new score, which will improve our capacity to isolate patients at risk of CAP due to M. tuberculosis at the time of hospitalisation.

@ERSpublications

New scores developed from a subset of CDC risk factors predicts $M$. tuberculosis in patients hospitalised with CAP http://ow.ly/qf1F2

For editorial comments see page 10 .

Received: Jan 302013 | Accepted after revision: May 102013 | First published online: June 212013

Conflict of interest: Disclosures can be found alongside the online version of this article at www.erj.ersjournals.com

Copyright @ERS 2014 


\section{Introduction}

Tuberculosis (TB) is the seventh leading cause of death in the world, and among infectious diseases it is second only to HIV [1]. Pulmonary TB classically presents as a chronic pneumonia with $\sim 4$ weeks between symptom onset and the first health consultation [2]. It may also present acutely, in which case it is essentially identical to the presentation of a patient with community-acquired pneumonia (CAP) [3].

Mycobacterium tuberculosis is identified as the aetiological agent for a small proportion of hospitalised patients with CAP. In fact, guidelines for managing CAP in several countries recognise M. tuberculosis as a potential pathogen [4-8].

Identifying and isolating hospitalised patients with pulmonary TB, before a final diagnosis is established, is essential in preventing nosocomial transmission of TB and outbreaks of multidrug-resistant TB [9]. To this end, institutional adherence to international standards of TB control is important. Alas, there is evidence that institutions often lack infection control strategies and adequate environmental measures to prevent the transmission of TB $[10,11]$.

It remains the clinician's responsibility to make sure that patients suspected of having TB undergo respiratory isolation [12]. To identify these patients, clinicians and infection preventionists primarily rely on 22 risk factors established for patients with chronic pneumonia caused by $M$. tuberculosis. These factors were drafted by the US Centers for Disease Control and Prevention (CDC) [13-15]. However, there is little in the literature on the relevance and magnitude of these risk factors in predicting CAP due to M. tuberculosis.

The Community-Acquired Pneumonia Organization (CAPO) cohort study is a multicenter international study of adult hospitalised patients with CAP [16]. The database for the study contains data on $>7000$ patients and includes all the CDC risk factors. In addition, the aetiology of CAP for each patient in the study is investigated during the initial workup. In some of these cases, M. tuberculosis has been identified as the cause of CAP. Our team's access to this unique dataset provided us the opportunity to evaluate the role of CDC risk factors for TB in adults hospitalised with CAP both with and without TB.

We conducted this study with the following objectives: 1) to evaluate the role of the CDC TB risk factors in the identification of patients with CAP due to TB; and 2) to identify the subset of CDC TB risk factors that best predict the presence of CAP due to TB.

\section{Study population and methods \\ Study design}

This was a secondary data analysis of the CAPO international cohort study database. Investigators completed a case report form for each case, which was subsequently transferred to the University of Louisville Division of Infectious Diseases Clinical and Translational Research Support Center (CTRSC, Louisville, KY, USA; www.ctrsc.net) through the Internet. Members of the CTRSC validated the cases as they were entered to ensure data quality. A sample of the case report form is available at the study website (www.caposite.com). Information on the CAPO project has been published previously [16].

\section{Patient characteristics}

The study population consisted of adults hospitalised with CAP from March 2001 to December 2011 from 113 hospitals in 33 countries. We reviewed medical records of patients with a diagnosis of CAP, which was established by the admitting physician. Patients enrolled in the study were $\geqslant 18$ years old and met the criteria for diagnosis of CAP, which includes a new pulmonary infiltrate (within $24 \mathrm{~h}$ of admission) associated with at least one of the following: new or increased cough with/without sputum production, fever $\left(>37.8^{\circ} \mathrm{C}\right.$ or $\left.>100^{\circ} \mathrm{F}\right)$ or hypothermia $\left(<35.6^{\circ} \mathrm{C}\right.$ or $\left.<96^{\circ} \mathrm{F}\right)$, leukocytosis, left shift or leukopenia. Pneumonia was considered to be community acquired if patients had no history of hospitalisation during the 2 weeks prior to admission. A case of CAP was considered to be due to M. tuberculosis in patients with positive respiratory cultures for $M$. tuberculosis. There were no exclusion criteria.

\section{Measurements}

Of the 22 CDC risk factors for TB, we evaluated the following 20: 1) night sweats; 2) haemoptysis; 3 ) weight loss; 4) hoarseness; 5) HIV/AIDS; 6) history of positive purified-protein derivative test (PPD); 7) homelessness; 8) alcohol or drug abuse; 9) healthcare worker; 10) prior history of TB; 11) age >65 years; 12) community living; 13) recent exposure to TB; 14) silicosis; 15) end-stage renal disease; 16) gastrectomy; 17) diabetes; 18) $10 \%$ or less of ideal body weight; 19) other immunosuppressive state (cancer of the gastrointestinal tract, haematological disorders or long-term cortisone use); and 20) intestinal bypass. We removed two risk factors (cough and pulmonary infiltrate) because these were part of the inclusion criteria to the study. All patients were admitted to the hospital with a working diagnosis of CAP and were initially treated empirically with antibiotics for bacterial CAP. 
TABLE 1 Demographic and clinical characteristics of the patients

\begin{tabular}{lccc} 
& TB CAP & Non-TB CAP & p-value \\
\hline Subjects $\mathbf{n}$ & 60 & 6916 & \\
Age years & $40.7 \pm 16.9$ & $64.7 \pm 19.1$ & $<0.001$ \\
Male & $35(58.3)$ & $4171(60.3)$ & 0.76 \\
Cavitary lesion & $1(1.7)$ & $24(0.34)$ & 0.19 \\
Upper lobe infiltrate & $35(58.3)$ & $1485(21.0)$ & $<0.001$ \\
Pleural effusion & $10(16.7)$ & $1490(21.1)$ & 0.4 \\
\hline
\end{tabular}

Data are presented as mean \pm SD or $n(\%)$, unless otherwise stated. TB: tuberculosis; CAP: communityacquired pneumonia.

\section{Statistical methods}

Baseline patient characteristics are presented as frequencies with percentages for categorical variables, and means with standard deviations for continuous variables, stratified based on TB status. We performed bivariate analysis of categorical variables using Chi-squared or Fisher's exact tests for categorical variables and the Mann-Whitney U-test for continuous variables.

We calculated the relative risk, sensitivity and specificity for the diagnosis of TB for each of the 20 CDC TB risk factors. We also calculated the sensitivity and specificity of having at least one of the CDC risk factors significantly associated with TB. Next, we calculated risk for CAP caused by TB for all patients according to the two following scores: 1) CDC TB risk score, and 2) CAPO TB risk score.

\section{CDC TB risk score}

The CDC TB risk score is the name we designated for a score calculated by summing each of the CDC risk factors for each patient. This score ranged from 0 points (no risk factors present) to 20 points (all risk factors present).

\section{CAPO TB risk score}

To better elucidate which of the 20 CDC TB risk factors were predictive of TB aetiology in our patients, we used a multivariate Poisson regression model with robust error variance. We included in the model variables that were statistically significant $(\mathrm{p} \leqslant 0.05)$ on bivariate analysis. In order to avoid colinearity in this model, we combined the following variables into one variable: 1 ) prior history of TB; 2) recent exposure to TB; and 3) history of positive PPD. We also combined the weight loss and $10 \%$ or less of ideal body weight variables into one variable. In the final Poisson regression model, variables that were not statistically significant $(\mathrm{p} \leqslant 0.05)$ were deleted.

The CAPO TB risk score utilised only the subset of variables selected in the final multivariate regression model. For the CAPO TB risk score, we also included the infiltrate localisation (upper lobe versus nonupper lobe) variable. The CAPO TB risk score was defined as the sum of each of the identified variables.

\section{Comparison of CDC TB risk score and CAPO TB risk score}

These two scores were then subjected to receiver operating characteristic (ROC) curve analysis for comparison of their ability to predict CAP due to TB. Statistical differences in the ROC curves were calculated according to previously published methods [17]. Unless otherwise specified, we considered $\mathrm{p} \leqslant 0.05$ to be statistically significant. We performed statistical analyses with SAS version 9.3 (SAS Institute, Cary, NC, USA) and Stata version 10 (StataCorp, College Station, TX, USA).

\section{Results}

\section{Demographic information}

This study included 6976 patients admitted to the hospital with criteria for the diagnosis of CAP. The mean age for the study population was $59.5 \pm 17.5$ years, and $4206(60.3 \%)$ of them were male. Of the total patients included in the study, 60 were diagnosed with CAP due to TB. Table 1 shows the demographic and clinical characteristics of the patients. Patients were distributed among four CAPO world regions. For patients with data on world region available, the distribution was: Asia/Africa/Australia (nine TB CAP and 76 non-TB CAP); Europe (20 TB CAP and 2035 non-TB CAP); Latin America (19 TB CAP and 1837 nonTB CAP); and USA/Canada (12 TB CAP and 2122 non-TB CAP). 
TABLE 2 Accuracy and relative risk of the factors significantly associated with tuberculosis (TB) presenting as pneumonia

\begin{tabular}{|c|c|c|c|c|c|c|}
\hline & TB CAP & Non-TB CAP & Sensitivity & Specificity & Relative risk $(95 \% \mathrm{Cl})$ & p-value \\
\hline Age $<65$ years & 55 (91.7) & $3051(44.1)$ & 91.7 & 55.9 & $13.7(5.5-34.2)$ & $<0.001$ \\
\hline Night sweats & $18(30)$ & $298(4.3)$ & 30.0 & 95.7 & $9.2(5.4-15.9)$ & $<0.001$ \\
\hline HIV/AIDS & $15(25)$ & $436(6.2)$ & 25.0 & 93.7 & $4.8(2.7-8.6)$ & $<0.001$ \\
\hline Absence of diabetes & 56 (93.3) & 5721 (82.7) & 93.3 & 17.3 & $2.9(1.1-8.0)$ & 0.02 \\
\hline $10 \%$ or less of ideal body weight & $12(20)$ & $251(3.6)$ & 20.0 & 96.4 & $6.4(3.4-11.9)$ & $<0.001$ \\
\hline History of positive PPD & $3(5)$ & $88(1.3)$ & 5 & 98.7 & $4.0(1.3-12.5)$ & 0.04 \\
\hline Weight loss & $27(45)$ & $517(7.5)$ & 45 & 92.5 & $9.7(5.9-16)$ & $<0.001$ \\
\hline
\end{tabular}

Data are presented as $\mathrm{n}(\%)$, unless otherwise stated. CAP: community-acquired pneumonia; PPD: purified-protein derivative test.

\section{CDC TB risk score}

The following CDC risk factors had a positive significant association with a diagnosis of TB: 1) night sweats; 2) haemoptysis; 3) weight loss; 4) HIV/AIDS; 5) $10 \%$ or less of ideal body weight; 6) prior history of TB; 7) recent exposure to TB; and 8) history of positive PPD. Conversely, patients with age $>65$ years and diabetes had a significantly lower risk of having TB. Thus, we present the accuracy and relative risk of these two variables with an inverse code, i.e. the association of $\mathrm{TB}$ aged $<65$ years and absence of diabetes (table 2).

At least one of the risk factors significantly associated with TB was present in $59(98.3 \%)$ patients with TB and $6256(90.5 \%)$ patients without TB. The presence of at least one of the risk factors significantly associated with TB had a sensitivity of $98.3 \%$, specificity of $9.5 \%$, positive predictive value of $0.9 \%$ and negative predictive value of $99.8 \%$ for the diagnosis of TB. Thus, in a patient without any of the CDC risk factors, TB is unlikely.

\section{CAPO TB risk score}

The CAPO TB risk score consisted of the following predictive variables: 1) night sweats; 2) haemoptysis; 3 ) combined weight loss $/ 10 \%$ or less of ideal body weight; 4) combined prior history of $\mathrm{TB} /$ recent exposure to TB/history of positive PPD; and 5) upper lobe infiltrate localisation. We excluded age $<65$ years because it did not substantially change the model. Of the 60 patients with CAP due to TB, none had all five risk factors included in the CAPO TB risk score. Of the 6916 patients with CAP not due to TB, $28 \%$ had only one of the five risk factors included in the CAPO TB risk score.

The percentage of patients with CAP based on the CDC TB risk factors and the CAPO TB risk scores is shown in figure 1.

FIGURE 1 Percentage of patients with community-acquired pneumonia based on the number of Centers of Disease Control and Prevention (CDC) tuberculosis (TB) risk factors and the Community-Acquired Pneumonia Organization (CAPO) TB risk scores.

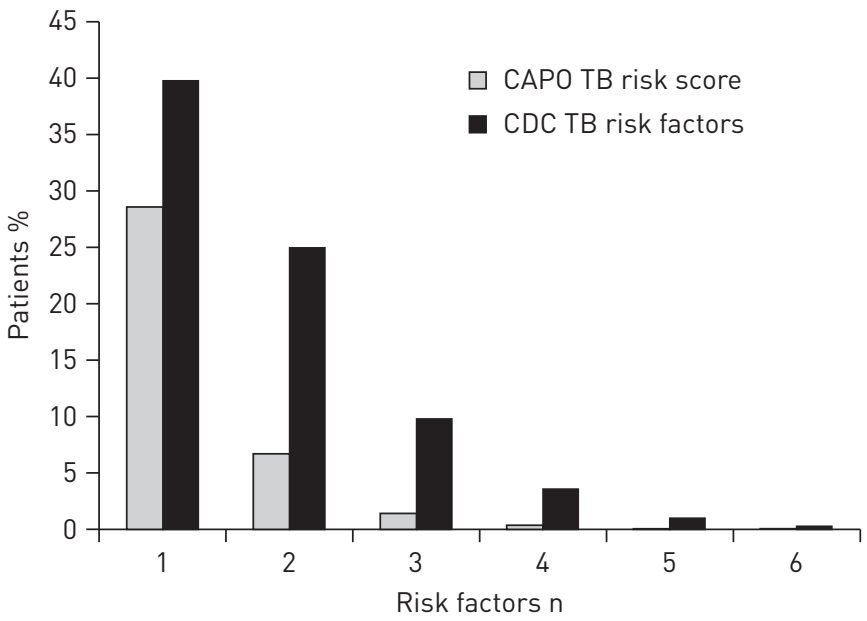




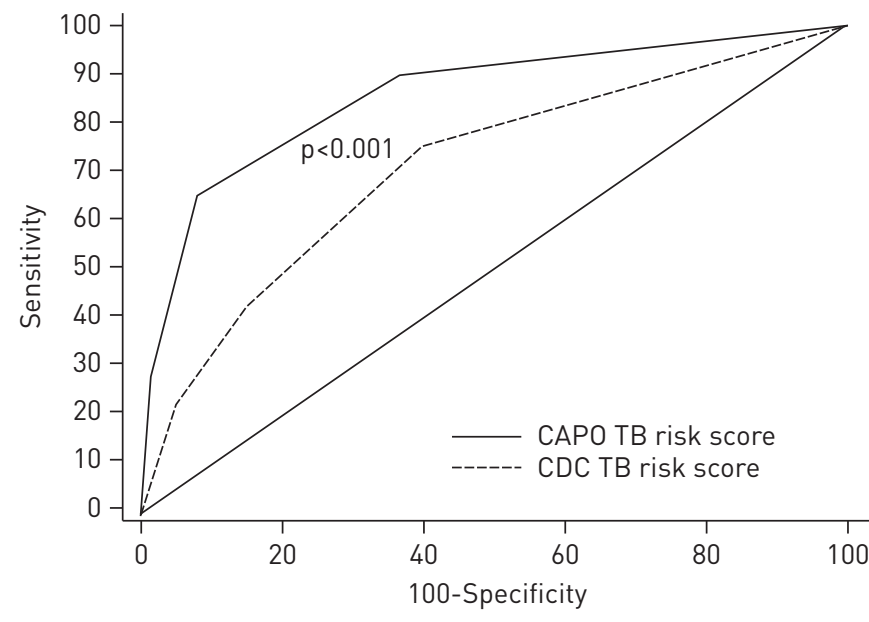

FIGURE 2 Receiver operating characteristic curves of two risk scores to predict tuberculosis (TB) in hospitalised patients with community-acquired pneumonia. CAPO: Community-Acquired Pneumonia Organization; CDC: Centers of Disease Control and Prevention.

\section{Comparison of scores to predict CAP due to TB}

The area under the ROC curve of the CAPO TB risk score $(0.89,95 \%$ CI $0.85-0.93 ; \mathrm{p}<0.001)$ was significantly higher compared with a model that included the CDC TB risk score as the predictive variable $(0.71,95 \%$ CI $0.64-0.78 ; \mathrm{p}<0.001)$. Figure 2 shows the ROC curves of the risk scores.

\section{Discussion}

This study indicates that most of the CDC TB risk factors have low sensitivity for predicting TB in hospitalised patients with CAP. Using a subset of CDC TB risk factors, as well as the presence of an upper lobe infiltrate, we have developed a simpler and more accurate prediction score: the CAPO TB risk score. This large dataset allowed us to perform robust statistical analyses and provide new findings to the preexisting literature.

This new score could be used as a tool for healthcare practitioners at the bedside for predicting the risk of $\mathrm{TB}$ in hospitalised patients with CAP.

Establishing the factors that are associated with an increased risk of TB in hospitalised patients with CAP is important for several reasons. First, recognising these factors in hospitalised patients should prompt respiratory isolation to prevent nosocomial transmission of TB. Outbreaks in healthcare settings have been associated with delayed diagnosis of TB disease and inadequate airborne precautions [14]. A challenge for the prevention of nosocomial transmission of $\mathrm{TB}$ is that institutions do not always comply with international standards of TB control [9-12]. Secondly, patients with these factors should be scrutinised for M. tuberculosis infection to allow a more timely diagnosis of TB. Thirdly, if empirical antibiotics for CAP are to be administered, these factors suggest that preference should be given to antibiotics that do not decrease the yield of diagnostic tests for M. tuberculosis. In view of this, there is evidence that empiric treatment with fluoroquinolones leads to a delay in the onset of anti-TB treatment [18, 19].

When assessing the probability of TB in hospitalised patients with pneumonia, it is important to use a sensitive tool to minimise the consequences of missing a diagnosis of pulmonary TB. Applying the presence of at least one of the CDC TB risk factors as criteria for suspecting TB and initiating respiratory isolation, only one case of TB would have been missed in the cohort of almost 7000 hospitalised patients with CAP included in our study. However, such a highly sensitive tool results in low specificity, with $90.5 \%$ of the patients without $\mathrm{TB}$ receiving respiratory isolation and workup for $\mathrm{TB}$, which may be infeasible. Using Poisson regression with robust error variance, we created a risk score based on five variables with high discrimination value for diagnosing TB. This model compared favourably with a model that had the sum of CDC TB risk factors as the predictive value.

While only a small proportion of hospitalised patients presenting with CAP had a diagnosis of TB in our study population $(0.86 \%)$, this may not be the same in areas with high incidence of TB. For instance, in a cohort of 346 patients hospitalised for CAP in Malaysia, 17 (5\%) patients had pulmonary TB, and M. tuberculosis was the fourth most frequently identified pathogen [20].

Few other studies evaluated risk factors for predicting a diagnosis of TB in patients with pneumonia. GAETA et al. [21] performed a case-control study which included patients that presented to an urban emergency department with a clinical picture consistent with pneumonia. Their study included 50 patients with TB that were sex- and age-matched to 50 patients without TB. They found that the following factors were 
significantly associated with a diagnosis of TB: HIV infection, injection drug use, recent PPD conversion, prior history of TB, haemoptysis and chest radiography consistent with TB. They subsequently used these risk factors as criteria for respiratory isolation in a validation sample of 103 patients, 22 of whom had TB. They found that the use of any of the criteria for the diagnosis of TB provided a sensitivity of $96 \%$, specificity of $14 \%$, positive predictive value of $23 \%$ and negative predictive value of $92 \%$ for diagnosing TB. Unlike our study, GAETA et al. [21] did not attempt to perform multivariate analysis of the variables associated with TB. This may explain some difference in the variables used in our TB risk score and the criteria they used for respiratory isolation. For instance, although HIV/AIDS was a significant risk factor for TB on univariate analysis in our study, it was no longer significant on multivariate analysis.

In a study with a design similar to ours, LIAM et al. [20] evaluated clinical features that can distinguish TB from non-TB in 346 patients hospitalised with CAP; 17 of those patients had TB. The emphasis in that study was on physiological variables. The following features were significantly associated with a diagnosis of TB: duration of symptoms of $>2$ weeks before hospital admission, night sweats, upper lobe involvement in the chest radiograph, total white blood cell count on admission $\leqslant 12 \times 10^{9}$ cells $\cdot \mathrm{L}^{-1}$ and lymphopenia. Patients aged $<40$ years had a lower risk of TB. A distinguishing feature of the study by LiAM et al. [20] is that half of their patients with TB had disease duration of $>2$ weeks as compared with only $7 \%$ of patients with non-TB pneumonia. In that study, chronic duration was heavily weighted in the group with TB, making the comparison of CAP due to TB and non-TB less compelling.

While our study and that of other investigators [20,21] evaluated risk factors for a diagnosis of TB in a cohort of patients presenting with CAP, others have assessed clinical and radiological findings of tuberculous pneumonia (as opposed to cavitary TB) in a cohort of adult patients with TB. For instance, in a cohort of 2228 patients with TB in Brazil, 59 (2\%) patients presented with noncavitary pulmonary consolidation, which occurred predominantly in the upper lobes [22]. In another study of 16 patients with tuberculous pneumonia, consolidations occurred more evenly in the lung lobes, and these patients had more fever, less haemoptysis and shorter duration of disease as compared with patients with cavitary TB [23]. In TB patients from our study, only one had a cavitary lesion, and most lesions were located in the upper lobe. The predominance of noncavitary lesions in our patient population perhaps supports the notion that the absence of cavitation is more common when TB presents acutely as a result of primary infection, although this has been disputed by other investigators [24, 25]. Using a DNA fingerprinting technique, GENG et al. [25] established that the main determinant of TB radiographic appearance is the patient's immune status. Time from infection was not a predictor of radiographic appearance in multivariate analysis.

Age $>65$ years and diabetes are factors that recognisably increase the risk of TB. However, in our study these factors were protective against it. The association in our study might be explained by residual confounding. Also, this finding should be interpreted in the context of the study population. Our study population included only patients presenting with CAP. Older age and presence of diabetes are themselves risk factors for CAP in general $[26,27]$. Thus, it is conceivable that age $>65$ years and diabetes remain risk factors for TB but not when the comparison is made with patients with CAP due to other aetiologies. Furthermore, one could ponder that TB may have a less florid presentation in patients with diabetes, and thus it may be less likely to present as CAP in these patients. Finally, the elderly may have a different presentation due to a higher rate of reactivation disease or altered immune status. In this context, it has been shown that the young are more prone to TB due to recently transmitted infection [28].

Our study has a number of limitations. Because the study was retrospective and observational, clinicians did not use a structured research interview form when assessing their patients. This may have led to under- or overestimation of particular risk factors. There is also the risk of selection bias, if, for instance, some patients suspected of having TB were initially excluded from the study by investigators. While our overall study sample size was large, the number of patients who had TB was moderate, making the assessment of the more infrequent risk factors imprecise. Finally, the workup for TB in hospitalised patients with CAP is variable, and therefore we cannot exclude the possibility that some of the patients had CAP due to TB, received empirical treatment with fluoroquinolones and were then misclassified as having CAP not due to TB.

Our study also has several strengths. It is the first study to examine the CDC TB risk factors in hospitalised patients with CAP. This study is also multicentred and multinational, making the findings more generalisable. It has an overall large sample size compared with other studies assessing risk factors for TB in patients with pneumonia and it assesses more risk factors for TB than previous studies [20, 21$]$.

The available literature on the value of risk factors for diagnosing TB in hospitalised patients is still scarce. Future studies are needed to prospectively evaluate the clinical practice value of the risk factors found to be significantly associated with TB in our study, and the newly built regression model. 
In conclusion, our findings indicate that using the CDC risk factors for $\mathrm{TB}$ as a tool to isolate patients hospitalised with CAP will identify most patients with CAP due to $\mathrm{TB}$, but will unnecessarily isolate an unacceptably large number of patients. The newly built CAPO TB risk score has high accuracy for the diagnosis of TB and is a promising tool to help clinicians in the decision making of isolating hospitalised patients with CAP at risk for TB.

\section{References}

World Health Organization. Global Tuberculosis Report 2012. Geneva, WHO, 2012.

Sreeramareddy CT, Panduru KV, Menten J, et al. Time delays in diagnosis of pulmonary tuberculosis: a systematic review of literature. BMC Infect Dis 2009; 9: 91.

3 Ramirez JA. Community-Acquired Pneumonia: a Plan for Implementing National Guidelines at the Local Hospital Level. Philadelphia, Lippincott Williams \& Wilkins, 2003.

4 Mandell LA, Wunderink RG, Anzueto A, et al. Infectious Diseases Society of America/American Thoracic Society consensus guidelines on the management of community-acquired pneumonia in adults. Clin Infect Dis 2007; 44: Suppl. 2, S27-S72.

5 Lim WS, Baudouin SV, George RC, et al. BTS guidelines for the management of community acquired pneumonia in adults: update 2009. Thorax 2009; 64: Suppl. 3, iiil-iii55.

6 Mandell LA, Marrie TJ, Grossman RF, et al. Summary of Canadian guidelines for the initial management of community-acquired pneumonia: an evidence-based update by the Canadian Infectious Disease Society and the Canadian Thoracic Society. Can J Infect Dis 2000; 11: 237-248.

7 Alfageme I, Aspa J, Bello S, et al. Normativas para el diagnóstico y el tratamiento de la neumonía adquirida en la comunidad. Sociedad Española de Neumología y Cirugía Torácica (SEPAR) [Guidelines for the diagnosis and management of community-acquired pneumonia. Spanish Society of Pulmonology and Thoracic Surgery (SEPAR)]. Arch Bronconeumol 2005; 41: 272-289.

8 Corrêa Rde A, Lundgren FL, Pereira-Silva JL, et al. Diretrizes brasileiras para pneumonia adquirida na comunidade em adultos imunocompetentes - 2009 [Brazilian guidelines for community-acquired pneumonia in immunocompetent adults - 2009]. J Bras Pneumol 2009; 35: 574-601.

9 Raviglione M, Marais B, Floyd K, et al. Scaling up interventions to achieve global tuberculosis control: progress and new developments. Lancet 2012; 379: 1902-1913.

10 Migliori GB, Sotgiu G, D'Ambrosio L, et al. TB and MDR/XDR-TB in European Union and European Economic Area countries: managed or mismanaged? Eur Respir J 2012; 39: 619-625.

11 Sotgiu G, D'Ambrosio L, Centis R, et al. TB and M/XDR-TB infection control in European TB reference centres: the Achilles' heel? Eur Respir J 2011; 38: 1221-1223.

12 Migliori GB, Zellweger JP, Abubakar I, et al. European Union standards for tuberculosis care. Eur Respir J 2012; 39 : $807-819$.

13 American Thoracic Society. Targeted tuberculin testing and treatment of latent tuberculosis infection. MMWR Recomm Rep 2000; 49: 1-51.

14 Jensen PA, Lambert LA, Iademarco MF, et al. Guidelines for preventing the transmission of Mycobacterium tuberculosis in health-care settings, 2005. MMWR Recomm Rep 2005; 54: 1-141.

15 CDC NCHHSTP Division of Tuberculosis Elimination. Introduction to the Core Curriculum on Tuberculosis: What the Clinician should Know. Atlanta, CDC, 2011.

16 Ramirez JA. Fostering international multicenter collaborative research: the CAPO Project. Int J Tuberc Lung Dis 2007; 11: 1062-1065.

17 DeLong ER, DeLong DM, Clarke-Pearson DL. Comparing the areas under two or more correlated receiver operating characteristic curves: a nonparametric approach. Biometrics 1988; 44: 837-845.

18 Dooley KE, Golub J, Goes FS, et al. Empiric treatment of community-acquired pneumonia with fluoroquinolones, and delays in the treatment of tuberculosis. Clin Infect Dis 2002; 34: 1607-1612.

19 Asnis DS, Cherian S, Sun T, et al. Pulmonary tuberculosis presenting as community-acquired pneumonia. Clin Infect Dis 2002; 35: 1574-1575.

20 Liam CK, Pang YK, Poosparajah S. Pulmonary tuberculosis presenting as community-acquired pneumonia. Respirology 2006; 11: 786-792.

21 Gaeta TJ, Webheh W, Yazji M, et al. Respiratory isolation of patients with suspected pulmonary tuberculosis in an inner-city hospital. Acad Emerg Med 1997; 4: 138-141.

22 Moreira J, Fochesatto JB, Moreira AL, et al. Pneumonia tuberculosa: um estudo de 59 casos confirmados microbiologicamente [Tuberculous pneumonia: a study of 59 microbiologically confirmed cases]. J Bras Pneumol 2011; 37: 232-237.

23 Lee KM, Choe KH, Kim SJ. Clinical investigation of cavitary tuberculosis and tuberculous pneumonia. Korean J Intern Med 2006; 21: 230-235.

24 Marais BJ, Parker SK, Verver S, et al. Primary and postprimary or reactivation tuberculosis: time to revise confusing terminology? AJR Am J Roentgenol 2009; 192: W198.

25 Geng E, Kreiswirth B, Burzynski J, et al. Clinical and radiographic correlates of primary and reactivation tuberculosis: a molecular epidemiology study. JAMA 2005; 293: 2740-2745.

26 Jokinen C, Heiskanen L, Juvonen H, et al. Incidence of community-acquired pneumonia in the population of four municipalities in eastern Finland. Am J Epidemiol 1993; 137: 977-988.

27 Jackson ML, Neuzil KM, Thompson WW, et al. The burden of community-acquired pneumonia in seniors: results of a population-based study. Clin Infect Dis 2004; 39: 1642-1650.

28 Alland D, Kalkut GE, Moss AR, et al. Transmission of tuberculosis in New York City. An analysis by DNA fingerprinting and conventional epidemiologic methods. N Engl J Med 1994; 330: 1710-1716. 